\title{
Perceptions of general dental practitioners of a local secondary care service in restorative dentistry
}

\author{
K. J. Fairbrother, ${ }^{1}$ and F. S. A. Nohl, ${ }^{2}$
}

\begin{abstract}
Aim A major role of the hospital based secondary care service in restorative dentistry is to accept referrals in order to formulate treatment plans. The aim of this survey was to improve service provision at Newcastle Dental Hospital by establishing baseline quality perceptions from referring general dental practitioners (GDPs).

Method A postal questionnaire was sent to 393 randomly selected local GDPs, with a response rate of $67 \%$.

Results Results indicated great demand for advice and treatment which was higher than expected for temporomandibular joint problems. $42 \%$ of GDPs felt that treatment plans were not helpful and the period of waiting for a response following consultation too long. The reasons for this perception are discussed.

Conclusions The need for better communication between primary and secondary care was highlighted, as was the distinct preference of GDPs for hospital consultants rather than registered specialist practitioners to carry out specialist treatment.
\end{abstract}

major role of the hospital based secondary care referral service $\mathrm{A}_{\text {in restorative dentistry is diagnosis of dental disease and for- }}$ mulation of appropriate treatment plans to facilitate patient management by general dental practitioners (GDPs). ${ }^{1}$ Good communication with GDPs is pivotal to the success of this service. Newcastle Dental Hospital (NDH) is a major centre in the UK for the provision of restorative dental care. Though referrals are plentiful, no local information is available regarding the proportion of GDPs who use this resource or of their perceptions of service quality. Literature regarding restorative dental referrals is sparse. Three studies investigated number and type of referrals: two concerned primarily prosthodontic referrals, ${ }^{2,3}$ one related to conservative dentistry. ${ }^{4}$ Another study addressed consultants' opinions of the standard of referral letters, ${ }^{5}$ but no publications appear to have investigated opinions of referring GDPs regarding their perceptions of service quality. In its document $A$ First Class Service, Quality in the New $\mathrm{NHS}^{6}$ the government has proposed its strategy for improving quality, delivering greater quality consistency, and making better use of NHS resources. It is in response to this agenda that hospital based restorative dentistry will need to evolve.

With the establishment of specialist lists in restorative dentistry (and the so called mono specialties of endodontics, periodontics and prosthodontics) by the General Dental Council in the UK, the

\footnotetext{
${ }^{*}$ Specialist Registrar in Restorative Dentistry, ${ }^{2}$ Senior Registrar in Restorative Dentistry, Department Of Restorative Dentistry, Newcastle Dental Hospital, Richardson Road, Newcastle upon Tyne NE2 4AZ.

${ }^{*}$ Correspondence to K. J. Fairbrother

REFEREED PAPER

Received 19.05.99; accepted 15.10.99

(C) British Dental Journal 2000; 188: 99-102
}

NHS hospital referral service in restorative dentistry could find itself in a competitive market with the private sector for the provision of specialist advice and treatment. As gatekeepers to specialist care and perhaps future fundholders, GDPs need increasingly to be regarded as customers in a consumer led market. Services will need to be developed which better suit the needs of patients and GDPs both in terms of quality and accessibility.

The goal of this survey of GDPs working within health authorities (HAs) in the north east of England was to provide information to help improve the quality of the referral service in restorative dentistry at NDH. The survey also aimed to discover from GDPs, where and by whom they thought secondary care in restorative dentistry for their patients should be delivered.

\section{Method}

A pilot questionnaire was sent to thirty randomly selected GDPs in the geographic area served by NDH who were listed by the former Family Health Service Authorities (FHSAs). These areas were: North Tyneside, Northumberland, Newcastle, South Tyneside/Gateshead, Durham and Sunderland. Following return of the completed pilot questionnaires, minor modifications to question style were made. Modified questionnaires and reply paid envelopes were sent to a further 363 randomly selected GDPs representing approximately $60 \%$ of GDPs in each former FHSA (Fig.1). Anonymity of the respondents was assured. Questionnaires sought to elicit:

- Whether there were practice guidelines in place for making specialist referrals in restorative dentistry

- The perceptions of GDPs regarding the frequency of referrals for advice or treatment to the subspecialties of restorative dentistry (periodontics, conservative dentistry, endodontics, removable

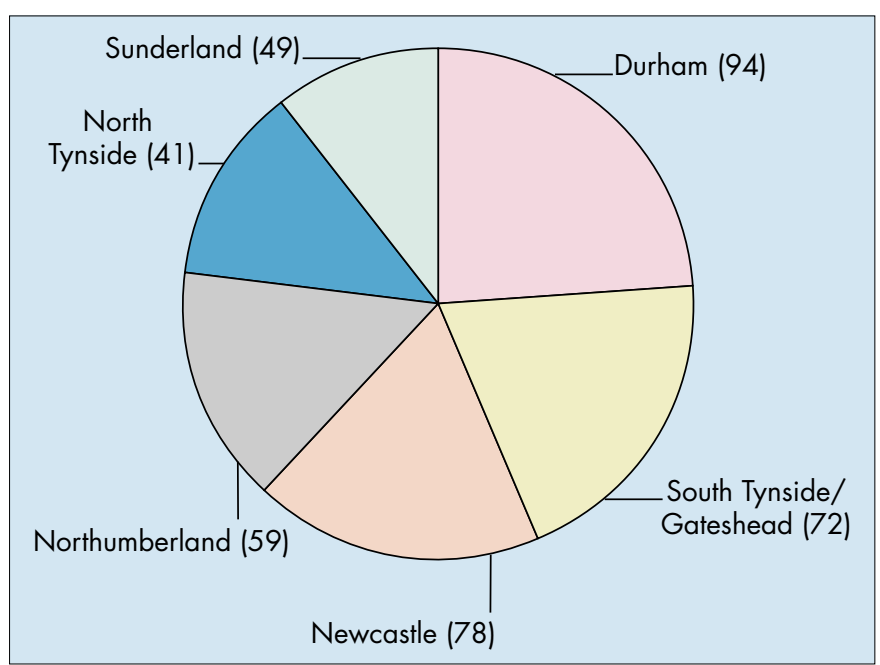

Fig. 1 Practitioners canvassed $(n=393)$ 
prosthodontics, temporomandibular joint (TMJ) related problems and implants)

- The opinions of GDPs on quality of service provided

- Any preference for whether hospital consultants or specialist practitioners should carry out specialist treatment.

In addition, respondents were encouraged to comment freely and asked to suggest ways in which improvements could be made. No attempt was made to follow up practitioners who failed to reply to the questionnaire.

\section{Results}

Of 393 questionnaires sent, 263 (66.9\%) were returned; similar proportions from each former FHSA canvassed (Fig. 2).

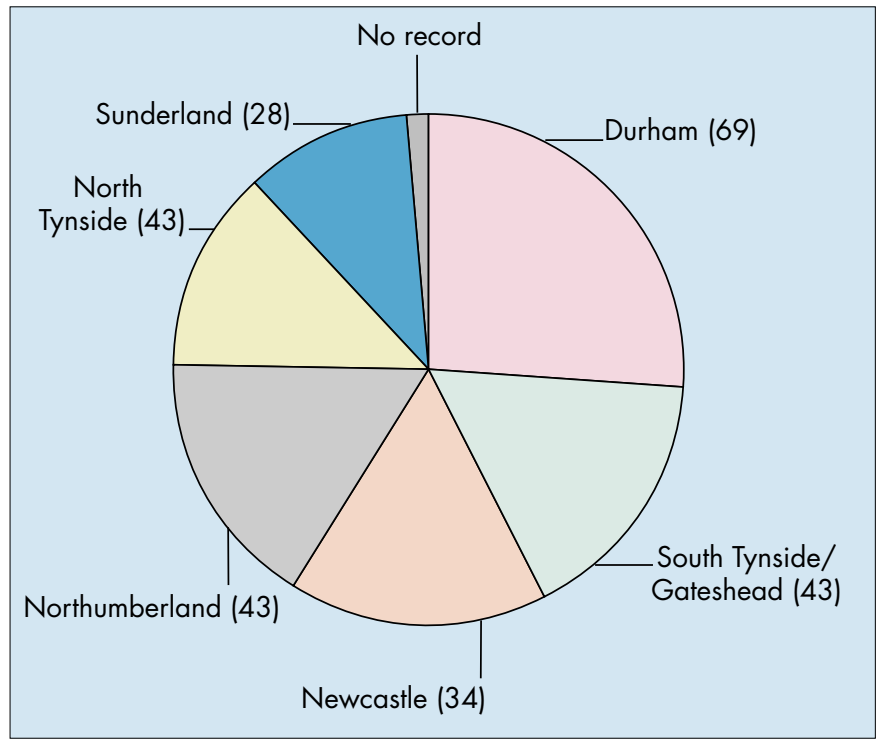

Fig. 2 Source of respondents $(n=263$ ).

Practice guidelines:

$70 \%$ of respondents had no guidelines for making referrals to the subspecialties of restorative dentistry. Nine per cent had guidelines and $21 \%$ of respondents gave no response to the question.

\section{Frequency of Referrals:}

Of 263 respondents 259 (98\%) perceived that they refer for advice and/or treatment at some time. Figure 3 shows the overall frequency of referrals for both advice and/or treatment. Thirty six per cent of respondents claimed to refer 1 patient per year for treatment and 32\% for advice. The most common frequency of referral was once per year. Figure 4 indicates the perceived frequency of referral for advice or treatment to each of the subspecialties. Regarding periodontics, 93\% of GDPs perceived that they referred at some time for treatment and $85 \%$ at some time for advice only. For conservative dentistry the pattern seen for periodontics was reversed: $88 \%$ for advice, $85 \%$ for treatment. The perceptions of referral pattern for prosthodontics and endodontics was similar to that for periodontics: (prosthodontics: $88 \%$ for treatment, $80 \%$ for advice, endodontics: $86 \%$ for treatment, $76 \%$ for advice). Approximately $46 \%$ of GDPs indicated that they referred for implant advice and treatment. $90 \%$ of GDPs perceived that they referred for treatment of TMJ problems in contrast to $82 \%$ for advice regarding management.

\section{Quality of Service.}

Figure 5 shows the attitudes of GDPs to treatment plans. Fifty eight percent of GDPs felt that written responses to referrals were helpful and achievable within the constraints of general dental practice. However, $31 \%$ of GDPs felt that treatment plans were unrealistic and $11 \%$ felt

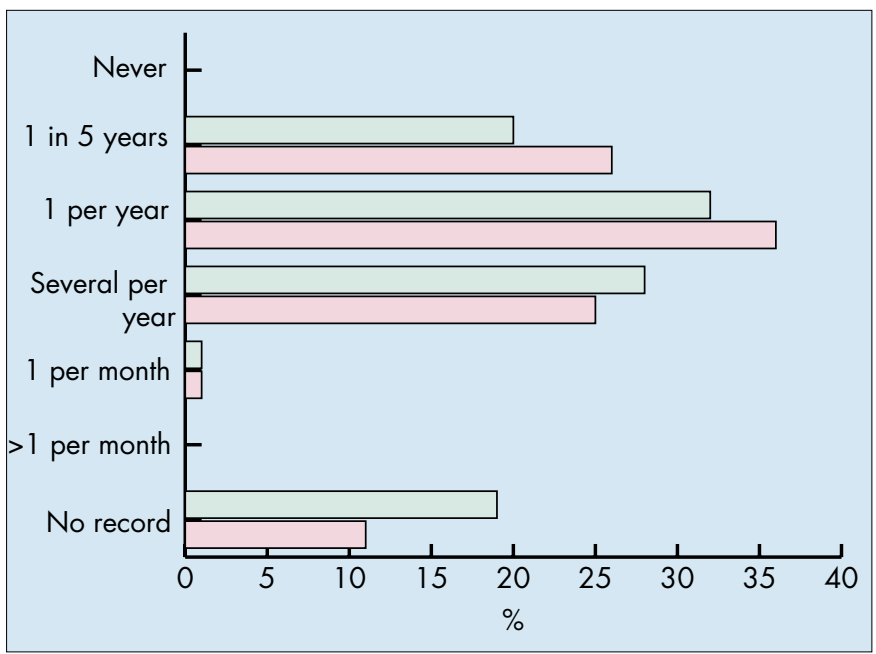

Fig. 3 Perceptions of frequency of all referrals for advice and treatment (advice: $\mathbf{n}=1139$, treatment: $\mathbf{n}=1224$ ). Red, treatment; green, advice.

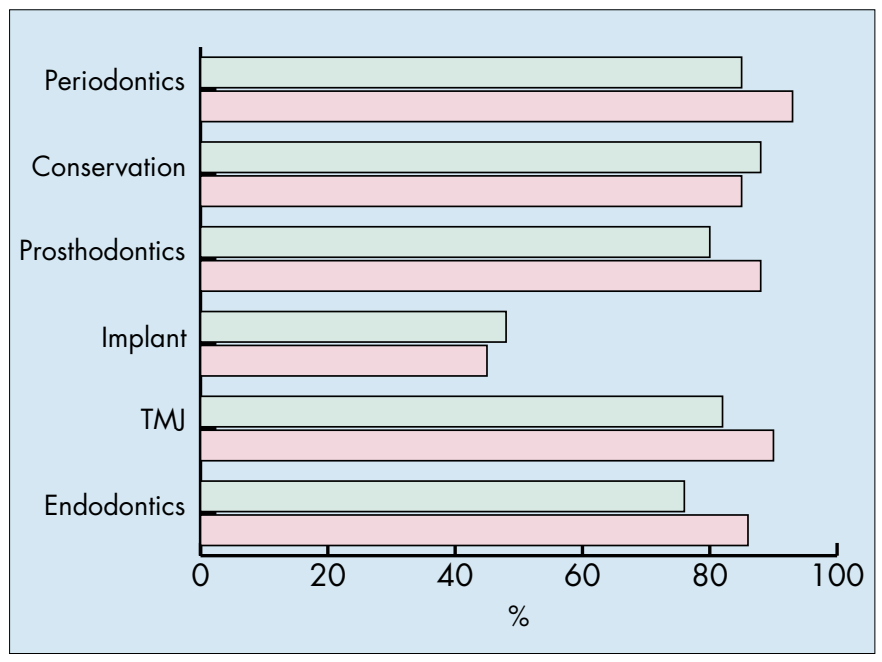

Fig.4 Requests for advice and treatment by specialty. Red, Treatment; green, advice.

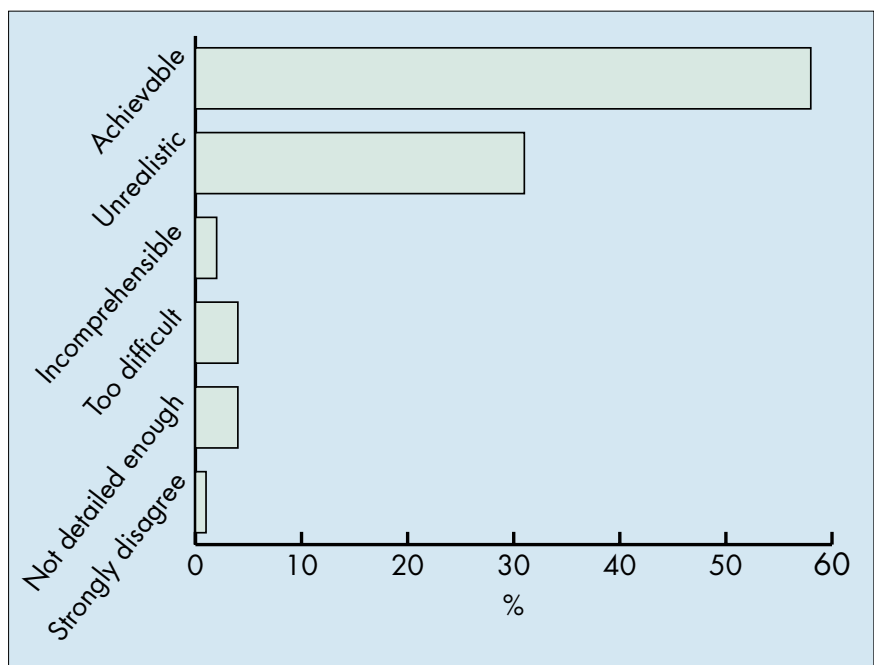

Fig. 5 Attitudes towards treatment plans received by general dental practitioners $(n=263)$. 


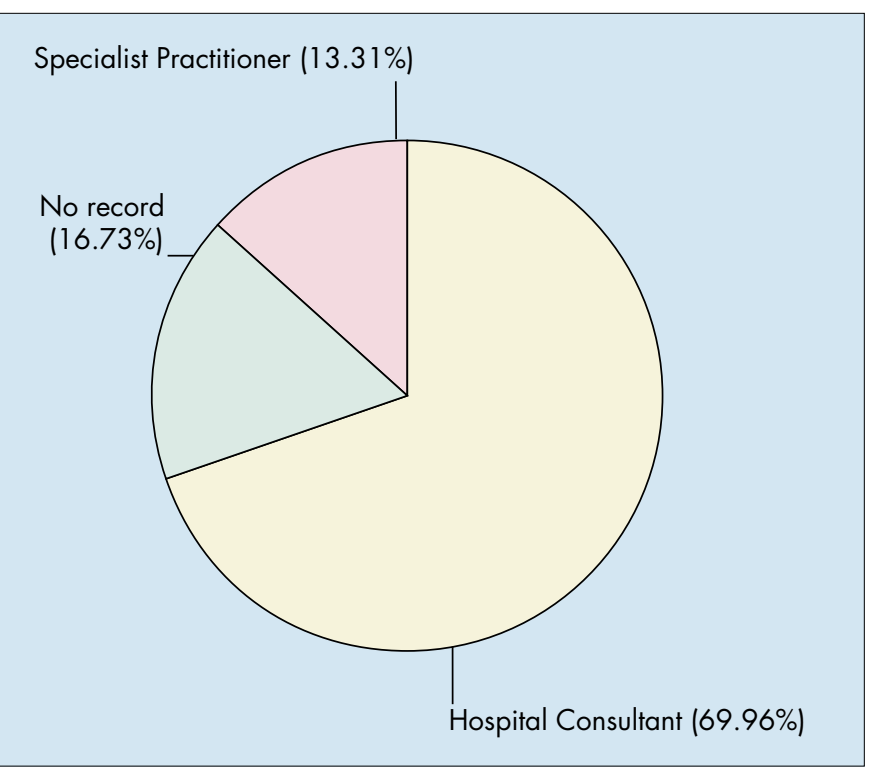

Fig. 6 Preferences of General Dental Practitioners for who should carry out specialist treatment $(n=263)$.

treatment plans were either unhelpful, incomprehensible or too difficult to accomplish. Eighty five per cent of GDPs indicated that an acknowledgement of receipt of referral would be desirable. Fifty four per cent felt that one month between referral and written response following consultation was a reasonable time period, whilst $31 \%$ felt two months was reasonable. Seventy seven per cent of GDPs stated a preference for treatment options rather than a single treatment plan.

\section{Treatment: Hospital Consultant or Registered Specialist} Practitioner?

Figure 6 shows preferences for who should carry out specialist treatment. Approximately $70 \%$ preferred a hospital consultant, $13 \%$ a specialist practitioner.

Free comments from practitioners were too broad to categorise individually. Pertinent comments are referred to in the discussion.

\section{Discussion}

The return rate of questionnaires $(66.9 \%)$ was high. Tan and Burke ${ }^{7}$ found an overall return rate of $61.7 \%$ in a study of a variety of questionnaires sent to GDPs. This finding suggests that the survey addressed key issues.

It is important to recognise that referral pattern responses were likely to be estimates rather than accurate records. It is also quite likely that outcomes of recent referrals would influence quality perceptions rather than experiences gained by GDPs over a period of time. However, the perceived level of referral (between one and eleven patients per year) is very similar to that found by Callis et al. ${ }^{4}$ where mean referral rate was 1.6 patients per dentist per year.

Similar return rates from GDPs in different former FHSAs suggests that the survey represents the feelings and attitudes of GDPs within the area served by NDH, and may be representative of GDPs nationally. The discrepancy between the number of questionnaires sent and received from the former North Tyneside FHSA may be due to uncertainty in completing this part of the questionnaire.

That $70 \%$ of practitioners had no guidelines for making referrals may not be surprising. What was of interest was that $9 \%$ of GDPs claimed to have practice guidelines in place for making specialist referrals. No attempt was made in the questionnaire to elucidate what these guidelines were. It is hoped that the development and wide distribution of referral guidelines, such as outlined in a document published by the Royal College of Surgeons of England (National Clinical Guidelines $^{8}$ ) would result in more appropriate referrals with the potential for reductions in waiting times and cost savings.
Apart from genuine need, the high demand for the referral service in restorative dentistry (98\% for advice and/or treatment at some time) may have other explanations:

- Some GDPs may acquiesce to requests for referral on financial grounds: patients either not being able, or not willing to pay for treatment proposed in general practice, (hospital treatment at the NDH is currently free of charge).

- Occasionally referrals may be made following a failed course of treatment, the patient and/or practitioner being unwilling to stand the extra cost of a repeat course. Referrals made on financial grounds would tend to undermine the principle of equity of access.

- From the free comments section of the questionnaire, it was evident that many practitioners used the referral service to 'off load' patients with difficult, non-dental management problems, for example where the dentist/patient relationship has broken down. This type of referral behaviour has been documented before. McAndrew ${ }^{5}$ noted that in many instances GDPs tended to refer patients they were not prepared to treat rather than those who definitely required specialist treatment. O'Brien et al. ${ }^{10}$ in a prospective investigation of orthodontic referrals, showed no significant association between treatment need and referral rate. The type of referrals alluded to obviously poses a problem for the profession and it would be helpful if the underlying reason for referral, albeit rather delicate, could be judiciously acknowledged from the outset. This approach may facilitate appropriate assistance and avoid unnecessary use of resources.

GDPs perceived that they referred more for treatment than advice with the exception of conservative dentistry and implants. This distribution is mirrored by other studies ${ }^{3,4,5}$ and may be explained by the following:

- GDPs feel more comfortable carrying out conservative dentistry as this reflects their skill base.

- GDPs are not happy with the fee scale structure within the NHS General Dental Services (GDS). Certain items of periodontic, prosthodontic and endodontic treatment (especially re-treatments) are perceived by some GDPs to be under-remunerated. A recent study by Linden ${ }^{8}$ highlighted the dissatisfaction of GDPs working within the constraints of the GDS regarding their desire and ability to treat periodontal disease.

The low perceived level of referral for advice and treatment using implants (48\% for advice) was surprising given the generally acknowledged awareness of GDPs and patients of the benefits of this treatment modality and may be explained by a variety of factors:

- A lack of awareness amongst GDPs of the potential for treatment with implants, perhaps indicating a need for continuing education in this area.

- Knowledge among practitioners that a strict protocol for accepting patients for treatment is operated by NDH.

- GDPs are aware of the high initial costs involved in treating patients with implant systems and are limiting the demand for such treatment at source.

- It is conceivable that a number of patients are referred to independent practitioners who offer implant treatment. However the demand for all other services is high.

There was an unexpectedly high perceived level of referral to the TMJ clinic. This matched referrals for periodontal treatment. A similarly high level of demand was reported in the study by Callis et al. ${ }^{4}$ There may be important ramifications for clinical resources and expertise in this area. Although our results relate to referrals to a dental teaching hospital it is conceivable that a consultant in restorative dentistry working in a district general hospital would 
expect to have as many TMJ as periodontal referrals. There are implications for other services, such as physiotherapy, if this perception is matched by reality.

Although $58 \%$ of GDPs felt that treatment plans were helpful and achievable within the constraints of the GDS, this left $42 \%$ of GDPs who felt that written responses to their referrals were not helpful. Of these, the majority felt that the treatment plan being proposed was unrealistic within the constraints of the GDS. Reasons were likely to be that treatment plans:

- Were too expensive.

- Were too difficult.

- Were not detailed enough.

- Recommended unfamiliar techniques. This may indicate a need for helping GDPs by further developing continuing education programs. Proposals by the General Dental Council ${ }^{11}$ for continuing education credits and re-certification aim to encourage lifelong learning.

In addition:

- Some GDPs strongly disagreed with proposed treatment plans.

- Some treatment plans were incomprehensible.

- Some GDPs stated they thought consultants were "out of touch with reality" and were providing treatment plans not achievable in general practice. It may be helpful for consultants to know whether or not the patient is likely to be treated under the NHS contract on returning to the referring practitioner. If advice to GDPs is to be helpful, consultants surely have a responsibility to familiarise themselves with the current NHS contract, regulations and financial constraints and to tailor treatment plans accordingly. A mandatory period spent in general dental practice as part of general professional training for those planning on a hospital career would go some way to addressing this problem.

It was clear that practitioners wished for a fast service; many GDPs (54\%) preferring a period of only one month between referral and written response. In view of high demand for the service this is unlikely to be practical except for urgent cases.

The large proportion of GDPs who responded that they would prefer a hospital based consultant rather than a specialist practitioner to carry out specialist treatment may be explained by:

- Cost of treatment. Specialist practitioners are likely to operate in a sector which will be independently financed. Costs to patients (or other third parties) are likely to be higher than fees currently being charged by GDPs working within the NHS. The perception may be that hospital consultants will provide a service at no cost to the patient, as has been the case to date.

- Competition. There may be a fear that patients sent to a specialist practice would never return to the referring dentist. Care would have to be exercised by specialist practitioners to ensure that patients are sent back to the referring GDP at the conclusion of specialist treatment.

In the free comments section, many GDPs indicated they were unhappy that their patients were not always seen by the consultant in person but by junior staff. There may be a lack of understanding within the practice community regarding training and manpower resources in the hospital service. If this misunderstanding exists, the onus is on the hospital service to inform GDPs of the situation.

It is clear from this survey that GDPs in the North East of England perceive the need for a hospital based referral service in restorative dentistry: the level of demand is high, not only for advice but for treatment in all aspects of the specialty. Moreover it would seem that in many cases GDPs felt the service should be expanded so that patients can be seen more quickly and specialist treatment performed more readily, preferably by a hospital consultant. There are a number of quality issues which need to be addressed. GDPs felt that written responses occasionally took too long to arrive and were not always aimed at the needs of a practitioner in the GDS. There needs to be closer liaison between all interested parties to develop referral guidelines and create a first class quality service for patients and their GDPs.

The authors would like to thank Mr DG Smith and Mr DJ Jacobs, Consultants in Restorative Dentistry for their help in preparing this paper, and the Clinical Audit Department of the Royal Victoria Hospital, Newcastle upon Tyne for their help with data collection.

1 A statement by the Consultants in Restorative Dentistry Group. Role of the Consultant Restorative Dentist in the General Hospital Service. $\mathrm{Br}$ Dent J 1983; 154: 181-182.

2 Yemm. R. Analysis of patients referred over a period of five years to a teaching hospital consultant service in dental prosthetics. Br Dent J 1985; 159: 304-306.

3 Basker R M, Harrison A, Ralph J P. A survey of patients referred to restorative dentistry clinics. Br Dent J 1988; 164: 105-108.

4 Callis P D, Charlton G, Clyde J S. A survey of patients seen in consultant clinics in conservative dentistry at Edinburgh Dental Hospital in 1990. Br Dent J 1993; 174: 106-110.

5 McAndrew R, Potts A J, McAndrew M, Adams S. Opinions of dental consultants on the standard of referral letter in dentistry. Br Dent J 1997; 182: 22-25.

6 The NHS Confederation. A First Class Service - quality in the new NHS. NHS Confederation 1998. Birmingham.

7 Tan R T and Burke F J T. Response rates to questionnaires mailed to dentists. A review of 77 publications. Int Dent J 1997; 47: 349-354.

8 Faculty of Dental Surgery. National Clinical Guidelines 1997. The Royal College of Surgeons of England.

9 Linden G J. Variation in periodontal referral by general dental practitioners. J Clin Periodontol 1998; 25: 655-661.

10 O'Brien K, McComb J L, Fox N, Bearn D and Wright J. Do dentists refer orthodontic patients inappropriately. Br Dent J 1996; 181: 132-136.

11 General Dental Council. Performance Review Scheme for the Dental Profession - A Consultation Paper. 1998.

\section{Submitting illustrations to the BDJ}

Authors submitting manuscripts for publication in the BDJ are reminded that two copies of all illustrations must be supplied. This will assist in speeding up the refereeing process manuscripts and illustrations are always sent to referees and a set of illustrations is required at the editorial office for reference. If you choose to submit $35-\mathrm{mm}$ transparencies then one set of these and one set of prints (colour or black \& white) will be acceptable.

We strongly advise authors to keep copies of all illustrations submitted in case letters are lost or damaged in transit. Please do not send glass-mounted transparencies - even when securely wrapped the glass will often arrive cracked or shattered, resulting in damage to the surface of the slide which usually renders it unsuitable for reproduction. 